\title{
Research on entropy properties comparison of different one-dimensional chaotic maps
}

\author{
HU Xing-hua ${ }^{a}$ GAO Lei-fu ${ }^{\text {b }}$ \\ College of science, Liaoning Technical University ,Fuxin,123000.China \\ a121681692@qq.com, ${ }^{\mathrm{b}}$ gaoleifu@163.com
}

\begin{abstract}
Chaos is the uncertain phenomenon of certain systems in evolution, while entropy, as the best quantization parameter of uncertain property, is very important for entropy properties comparison of different one-dimensional chaotic maps.Compare entropy properties of different classical chaotic maps separately based on tent map,cubic map, unlimited folding map and Chebyshev map.The results show that when the system parameters are confirmed, the initial value hardly effects the entropy of chaos system. At the same time the longer the chaos sequence, the nearer the entropy value of chaos system to the theory limits. The system entropy is changing with the system value.Under the same condition, the entropy of tent map will get near to the theory limit faster while stability is stronger. This means the ergodic of tent map sequence is more uniform, which presents the theory base for that it is the best choice chaotic map practically.
\end{abstract}

Keywords: Chaos, entropy, chaotic map.

\section{Introduction}

Chaos is the complex phenomenon that comes from certain system because of inner feature. It is a fade random movement like random ${ }^{[1]}$. Chaos is not simply out of order, but no obvious features like period and symmetry in normal integer dimension. The referenced fractional dimensional space has abundant interior level ordered structure, which is a new style of nonlinear system. Because of its unique properties, as inner random, high sensitive to initial value, and ergodicity of phase space, Chaos is applied in many areas. Choi did the function optimization by introducing Chaos dynamic into steepest descent method ${ }^{[2]}$. Wang Zicai introduced the ergodicity of Chaos into simulated annealing algorithm ${ }^{[3]}$.Jiang introduced Chaos map into Particle Swarm Optimization ${ }^{[4]}$.Zhang Jinliang presented PSO-LSSVM based on Wavelet Transform and Particle Warm Optimization, and a complexed prediction method $^{[5]}$ of Chaos time sequence of LARCH.

All of the study above is for the uncertain feature of Chaos (ergodicity, random and so on) together with optimization, or to do prediction with modern mathematic methods. As the uncertain quantization parameter of Chaos system,Lyaponov exponent ${ }^{[6-7]}$,pace observation method, Poincare section method, entropy ${ }^{[8-9]}$, and fractal dimension ${ }^{[10]}$. The most important quantization parameter, at the same time most commonly used, is Lyaponov exponent. When it is $>0$,system is qualitatively is in Chaos, but it cannot be used in adjust the complexity of system. Other parameters can be used to do this, while entropy and fractal dimension can qualitatively be used. Pan Xinyu and so on studied the entropy properties of Logistic Chaos system ${ }^{[11]}$. Based on reference[11], this work went on studying the relationship between the properties tent map entropy and system initial value, parameter. Especially by comparing the entropy properties of different 1-dimension Chaos systems, by data analysis of the limit process of graduate entropies, this presents the reason why tent map has better distribution property from the uniformity.

\section{Analysis of entropy properties}

The entropy is a function of describing system state. The system entropy directly reflects the uniformity of state. Shannon,who first introduced the concept of entropy in Information Theory, named it as information entropy. That is for a uncertain system, if the state is described by random 
variable $X$, for discrete random variable,let $X$ be $X=\left\{x_{1}, x_{2}, \cdots, x_{n}\right\},(n \geq 2)$, each value's referenced probability is $P=\left\{p_{1}, p_{2}, \cdots, p_{n}\right\},\left(0 \leq p_{i} \leq 1\right)$, and $\sum_{i=1}^{n} p_{i}=1$, the information entropy is defined as $S=-\sum_{i} p_{i} \ln \left(p_{i}\right)$, whose essence describes that the complexity and randomness of any movement or system evolution. Factually $X$ can be stated as the state area of system studied.The value of $X$ can be selected as its different equal division,or a equal non-overlapped cover.It can be easily proved that when $X$ is equal probability distribution, when continuous it is uniformed distribution, the entropy function gets max value,which means the probability of $X$ in every equal quantity area,as when $p_{i}=1 / n,(i=1,2, \cdots, n)$, the entropy function has its max value $\max (S)=\max \left\{-\sum_{i} p_{i} \ln \left(p_{i}\right)\right\}=\ln n$.

\section{Entropy properties study of different Chaos systems}

All the experiment used Matlab 2015,in the environment of Intel(R), Pentium(R),Core(TM) i7-3520M CPU,2.9GHz,4.00GB,Windows7, no data deleted.

\subsection{Study of entropy properties of Chaos system}

\subsubsection{Study of entropy properties of tent map}

As the classical tent map,

$$
\begin{aligned}
& x_{n+1}=a-(1+a)\left|x_{n}\right|, \\
& -1<x_{0}<1,0<a<1, n=01,2, \cdots
\end{aligned}
$$

For different parameter $a$ and initial value $x_{0}$,analyze the sequence distribution of system evolution. The steps are,select the system initial value $x_{0}$ from -0.99 to 0.99 , one step is 0.02 , in total 100.The parameter $a$ is selected from 0.001 to 0.999 , step 0.002 , in total 500 . The discrete Chaos sequence $x_{n}$ from (1) is from [-1,1]. When statist sequence,divide [-1,1] into 20 intervals,step is 0.1 ,in another way 100 intervals with step 0.02, the more intervals, the more elaborate. If the length of Chaos sequence from (1) is $N$,statist the sequence number $n_{i}(i=1,2, \cdots 20)$ in every interval,so the statistic probability is $p_{i}=n_{i} / N(i=1,2, \cdots, 20)$, and from concept the referenced entropy is $S=-\sum_{i} p_{i} \ln \left(p_{i}\right)$,the theory extremum is $\ln 20=2.9957$, while if the number of intervals is 100 , $\ln 100=4.6052$.

Fig.1 presents for parameter $a=0.137$ and $a=0.995$,in the Chaos system (1) for different initial values, the length of sequence iterated is $N=100000$, the distribution of Chaos sequence by dividing $[-1,1]$ into 100 intervals. From Fig 1,different initial values effect the distribution of Chaos sequence

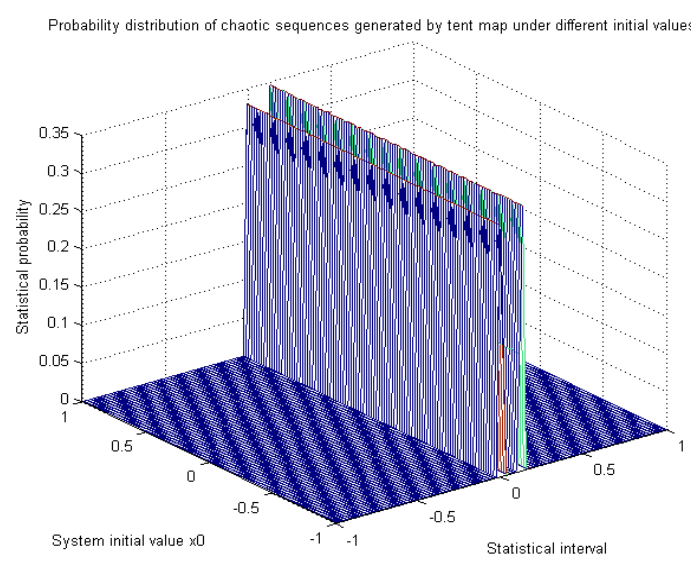

(a) parameter $a=0.137$

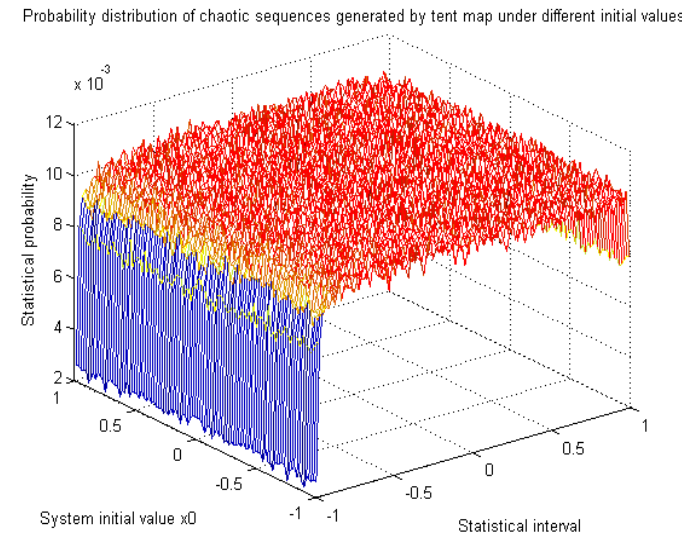

(b) parameter $a=0.995$

Fig.1 distribution of Chaos sequence with different initial values 
With parameter $a$ getting larger, the data range of Chaos sequence increases, and the distribution is becoming more and more uniformed, so that its entropy is bigger. Do many experiments with different parameters in order to get wide results. The representative results are as Fig 2 and 3.

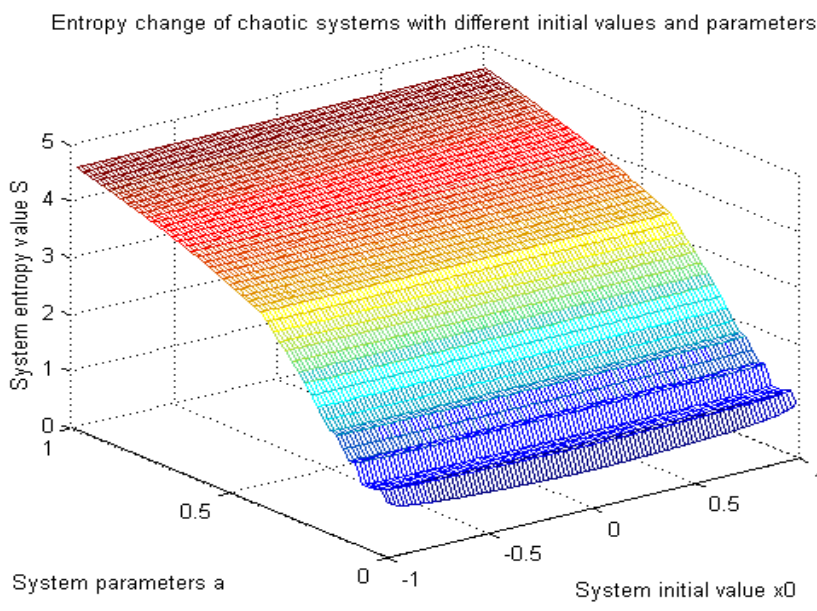

(a) distribution of Chaos system entropy

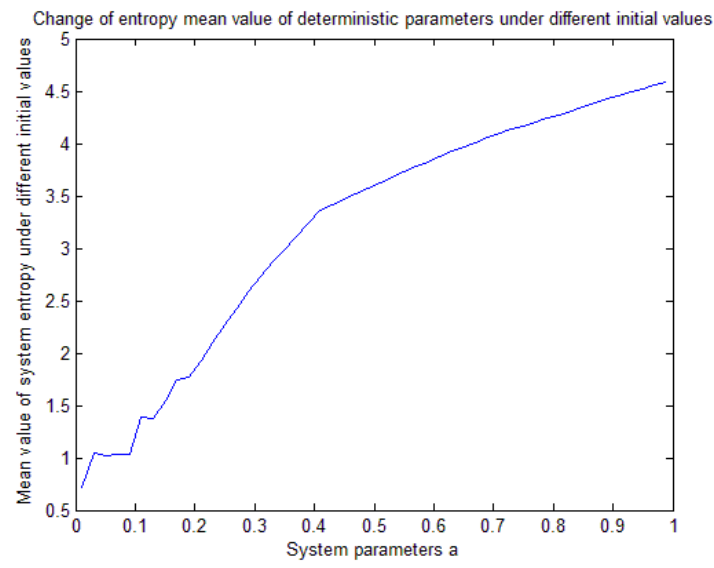

(b) average value distribution of Chaos entropy

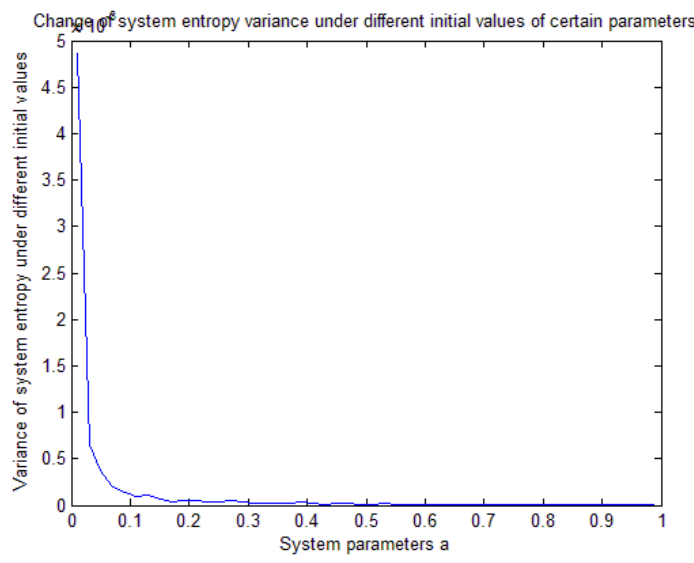

(c) variance distribution of Chaos entropy Fig.2 length of Chaos sequence 10000, number of interval division 100, analysis of effect of different initial values to entropy properties of Chaos system

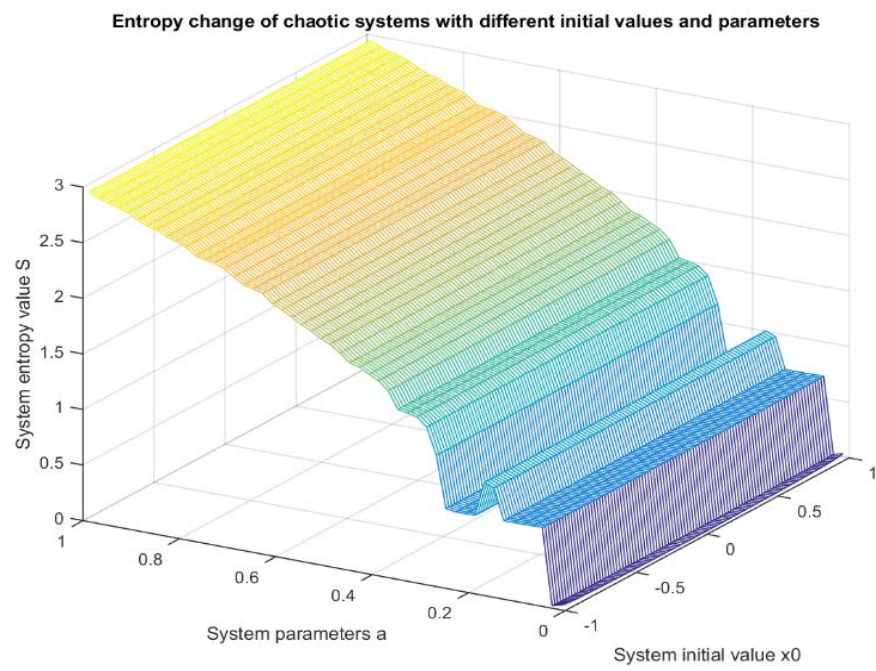

(a) distribution of Chaos system entropy 


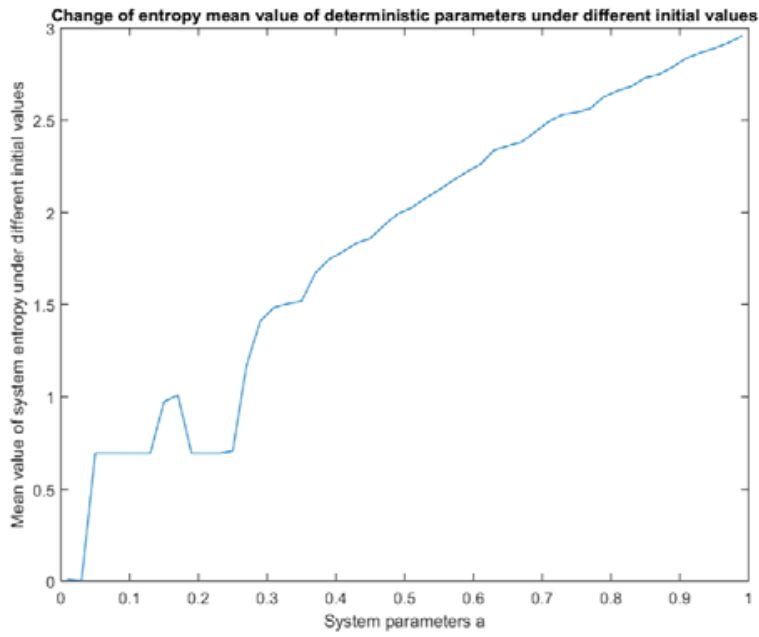

(b) average value distribution of Chaos entropy

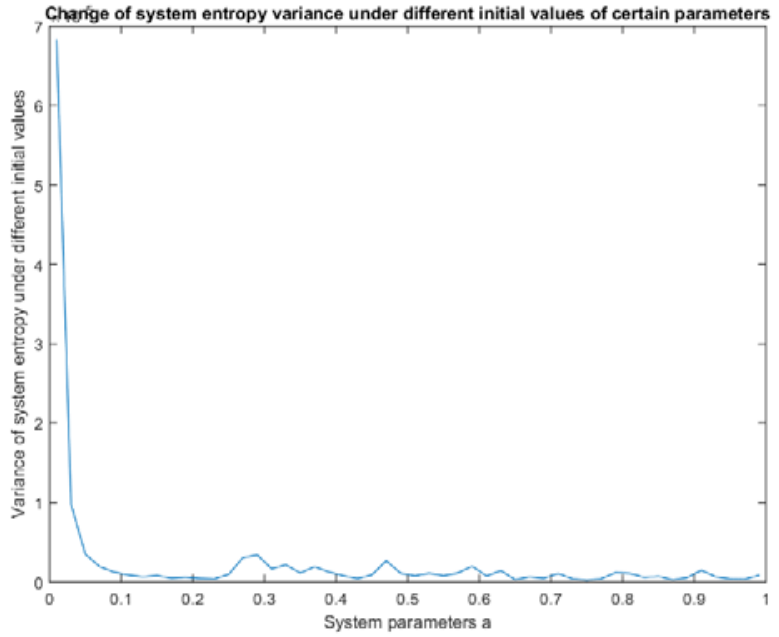

(c) variance distribution of Chaos entropy Fig.3 length of Chaos sequence 100000, number of interval division 20, analysis of effect of different initial values to entropy properties of Chaos system

From Fig 2, 3, and experiments, no matter the number of statistic is 20 or 100 or more, when the parameter $a$ is confirmed, no matter the length of data and different system initial values, the entropy value remains the same. When the parameter $a$ increases, the entropy increases. When $a$ gets near to 1, the entropy is the maximum 4.6002 and 2.9956, near to the theory limits separately. Of course there is some mutation points in the process of entropy increasing, which means for the special parameter $a$, the Chaos system (1) has not been in Chaos state, but stayed in the turnoff of multi-period, while the data around period points is too dense, all the data only stay in several statistic intervals, so that the entropy of these points gets smaller. For the comparison of Fig 2 and 3,enlarge the length of Chaos sequence, the statistic intervals get less, the amount of data in every statistic interval is more sufficient, the probability is more steady.The 3-D Fig 3 is more smooth than Fig 2, entropy variance Fig 2 is two order of magnitude smaller that Fig 2, which is also a good provement.

From the analysis of experiment results, the entropy of Chaos system (1) is basically decided by parameter $a$,not related to the initial value.On the view of statistic,the sequence distribution of the Chaos system with certain parameter $a$ has somehow regular pattern,some relative steady parts under the property of uncertain of Chaos system.At the same time the entropy of Chaos system (1) increases with $a$, in the end near to the theory limit.This means the sequence distribution of Chaos system (1) is more and more uniformed.

\subsubsection{Comparison of entropy properties of popular 1-dimensional Chaos system}

When the property of Chaos sequence is applied in other areas, the Chaos systems that always need studying are,

Cubic map

Infinite fold map

$$
x_{n+1}=4 x_{n}^{3}-3 x_{n},-1<x_{0}<1, n=01,2, \cdots
$$

$$
x_{n+1}=\sin \left(\frac{2}{x_{n}}\right),-1<x_{0}<1, n=0,1,2, \cdots
$$

Chebyshev map

$$
\begin{aligned}
& x_{n+1}=\cos \left(k \arccos x_{n}\right), \\
& -1<x_{0}<1, k \geq 2, n=01,2, \cdots
\end{aligned}
$$

For Chaos system (2)-(4),do experiments like 3.1.1.Based on normal parameters, for certain Chaos areas, first study different initial values effects different Chaos systems sequence probability, calculate entropy by the definition. Then consider when the length of Chaos sequence increase, how about the uniform of the probability distribution of every Chaos system? What is process of every entropy of Chaos system to the theory limit? As Fig 4 and 5, in different conditions, the sequences of 
Chaos system (1)-(4), Chaos map sequence probability distribution based on different length and interval divided. Fig 6 and 7 present the entropies.

Probability distribution of chaotic sequences generated by tent map under different initial values

Probability distribution of chaotic sequences generated by cubic map under different initial values

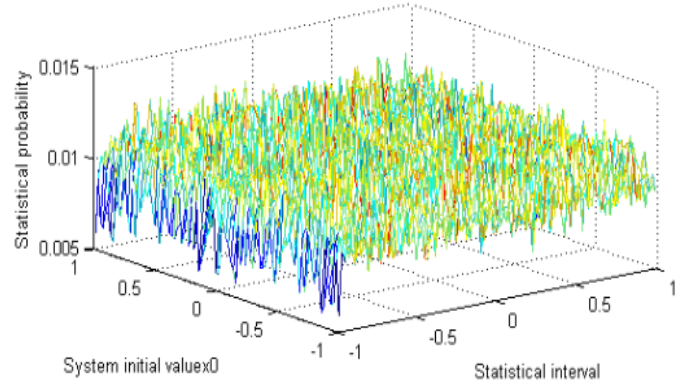

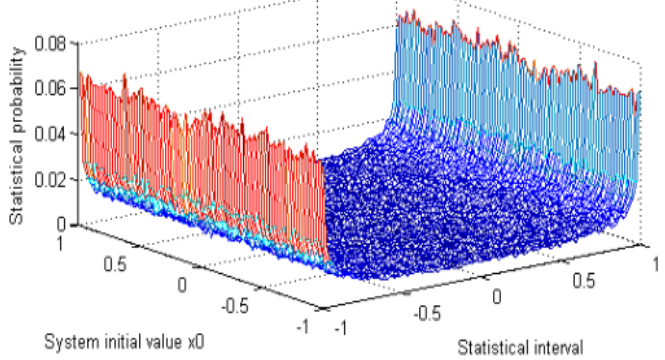

Statistical interval

'Trobability distribution of chaotic sequences generated by infinite folded map under different initial values Probability distribution of chaotic sequences generated by Chebyshev map under different initial values
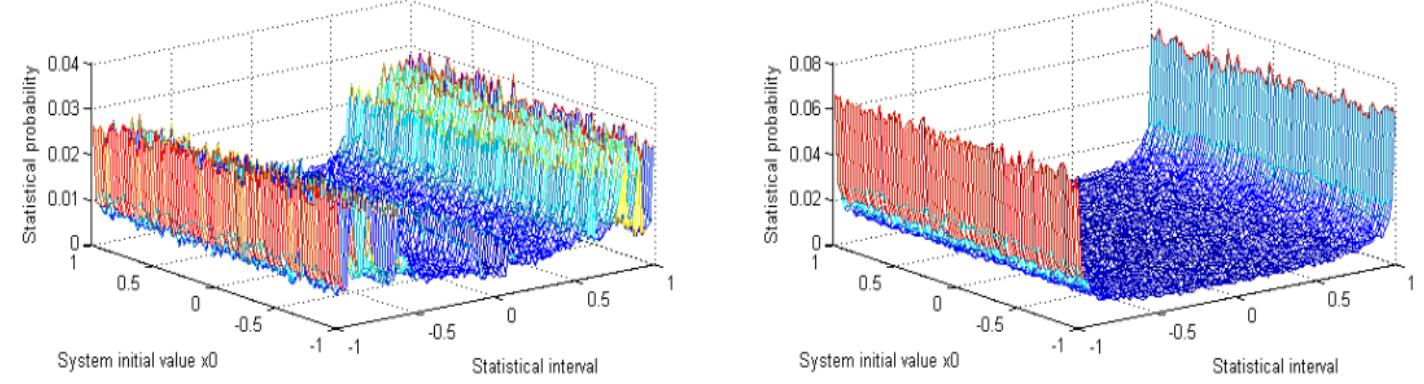

Fig.4 sequence length 10000, number of interval 100, Chaos sequence probability distribution of different initial values
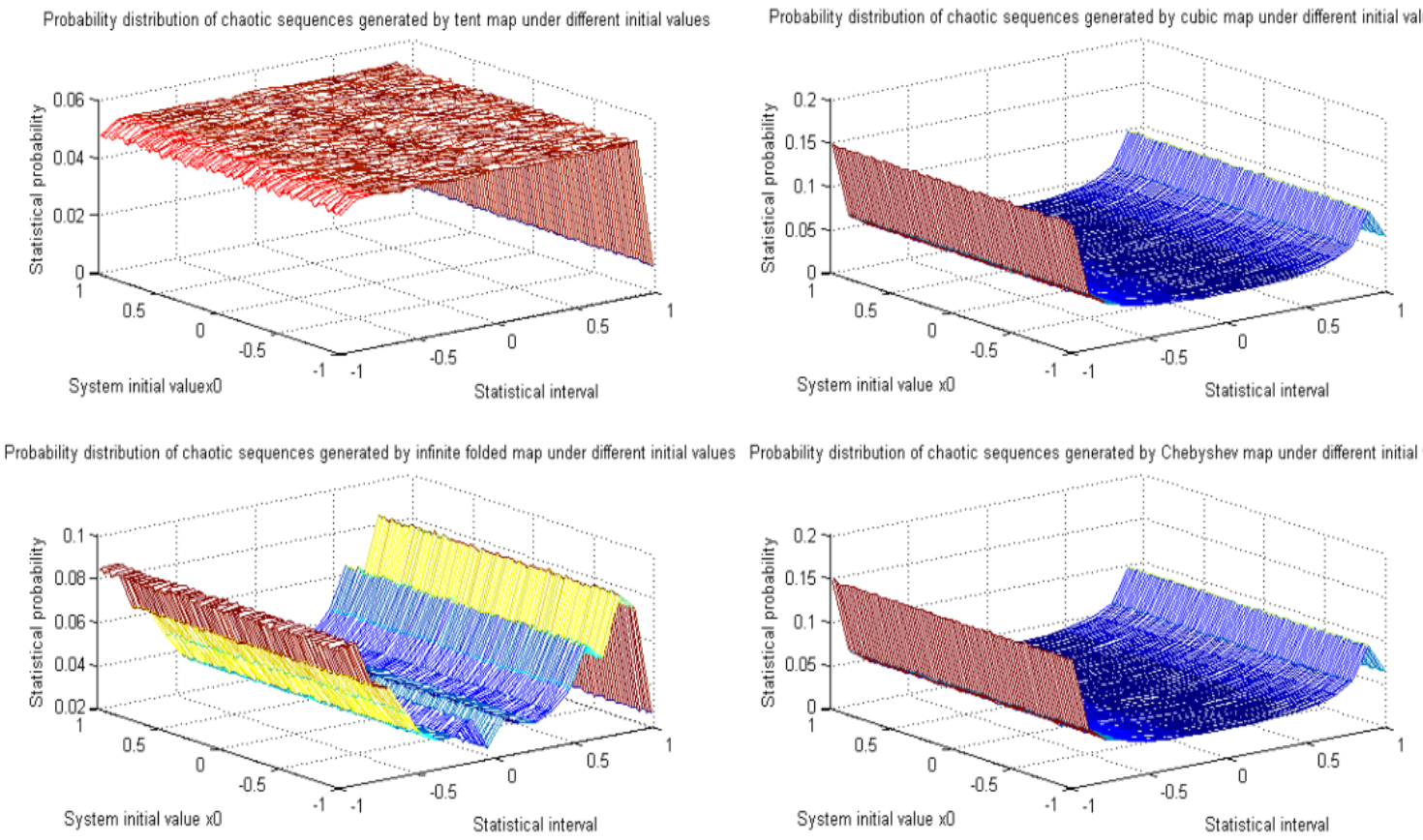

Fig. 5 Sequence length 100000, number of interval 20, Chaos sequence probability distribution of different initial values 

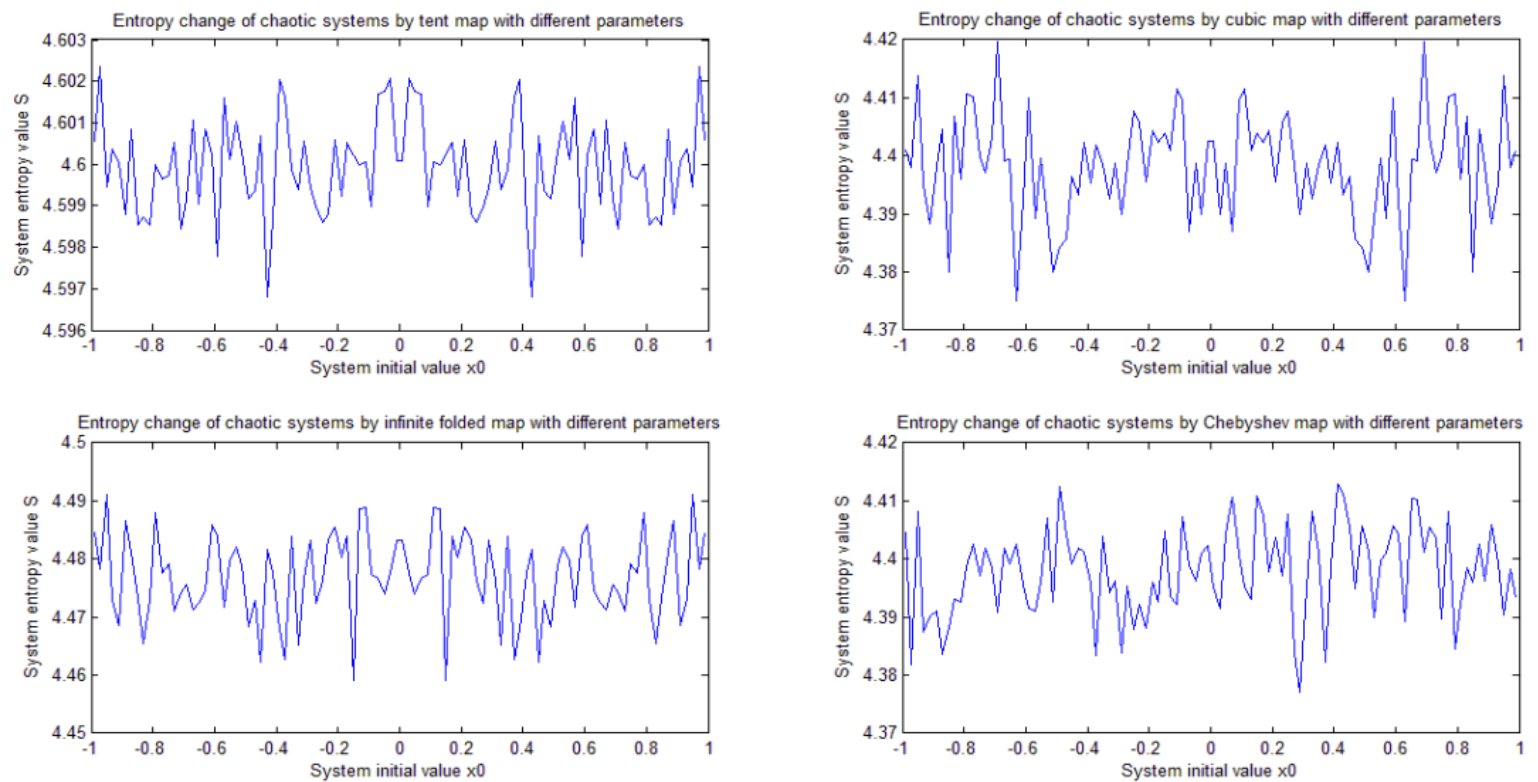

Fig.6 Sequence length 10000, number of interval 100, Chaos entropies with different initial values
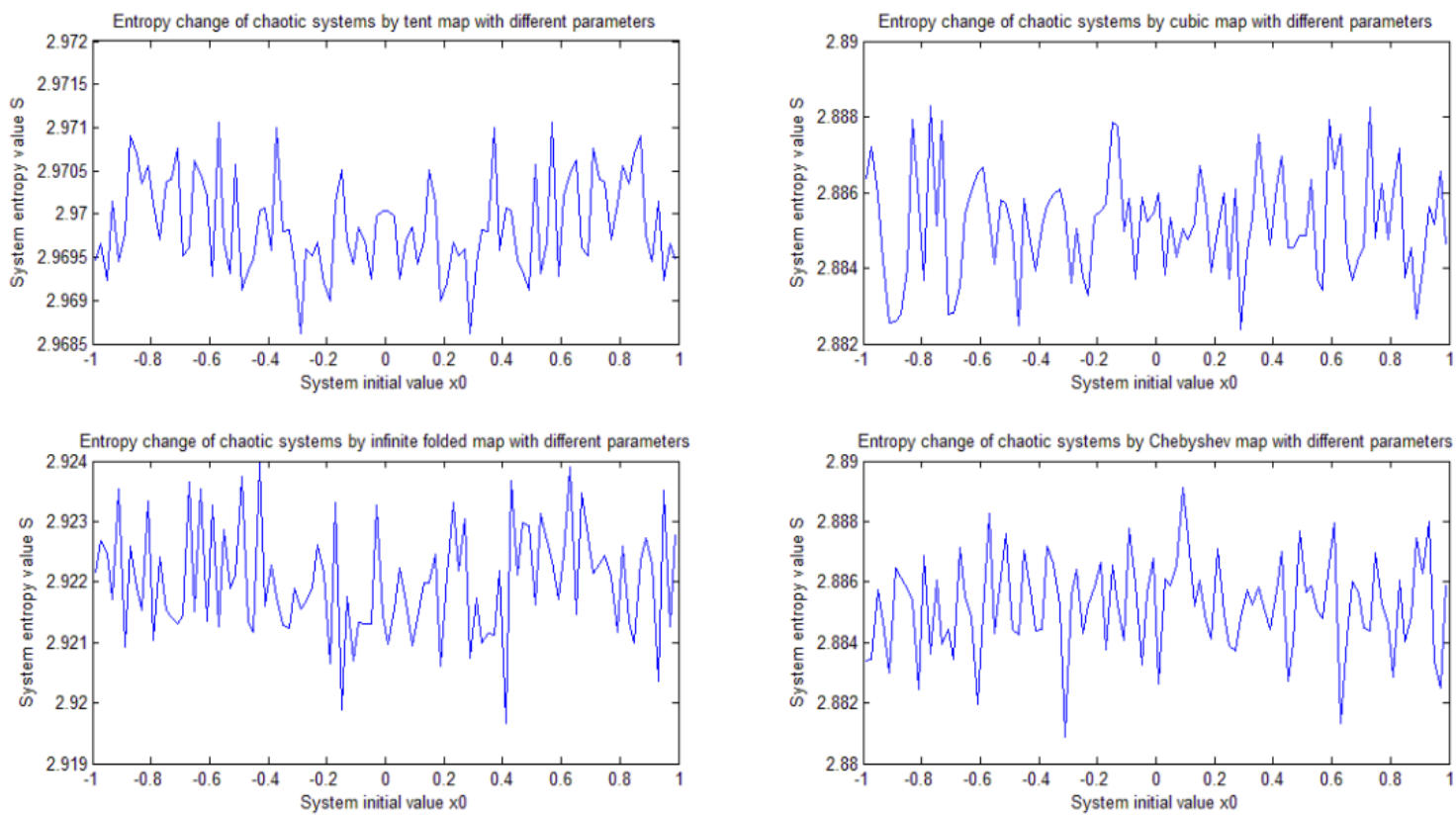

Fig.7 sequence length 100000, number of interval 20, Chaos entropies with different initial values

From Fig 4, 5, 6 and 7, the results show that the change of initial state (initial value) does not effect the entropy of Chaos system much. Besides, the longer the Chaos sequence, the nearer entropy of Chaos system to its theory limit, and the change is small. Table 1 and Table 2 gives the results in details. 
Table 1 average entropies of different Chaos under different initial values with sequence length

\begin{tabular}{c|ccccc}
\hline $\begin{array}{c}\text { Chaos system } \\
\text { aaverage of entrepy }\end{array}$ & $\begin{array}{c}\text { Tent } \\
\text { map }\end{array}$ & $\begin{array}{c}\text { Cubic } \\
\text { map }\end{array}$ & $\begin{array}{c}\text { Infinite fold } \\
\text { map }\end{array}$ & $\begin{array}{c}\text { Chebyshev } \\
\text { map }\end{array}$ & Theory entropy $\left(\begin{array}{l}\mathrm{M}=20 \\
\mathrm{M}=100\end{array}\right)$ \\
\hline \multirow{2}{*}{$N=10000$} & 2.9694 & 2.8845 & 2.9208 & 2.8847 & 2.9957 \\
& 4.5999 & 4.3983 & 4.4770 & 4.3978 & 4.6052 \\
$N=100000$ & 2.9699 & 2.8852 & 2.9220 & 2.8852 & 2.9957 \\
& 4.6043 & 4.4026 & 4.4825 & 4.4025 & 4.6052 \\
$N=1000000$ & 2.9701 & 2.8853 & 2.9220 & 2.8637 & 2.9957 \\
& 4.6047 & 4.4029 & 4.4828 & 4.3681 & 4.6052 \\
\hline
\end{tabular}

Table 2 entropy variances of different Chaos system with different initial values with sequence length

\begin{tabular}{|c|c|c|c|c|}
\hline Sequence length & Tent map & Cubic map & Infinite map & Chebyshev map \\
\hline \multirow[b]{2}{*}{$N=10000$} & $2.66 \mathrm{e}-06$ & 3.263e-05 & 7.11 e-06 & 2.808 e-05 \\
\hline & $1.37 \mathrm{e}-06$ & $8.224 \mathrm{e}-05$ & $5.484 \mathrm{e}-05$ & 6.036 e-05 \\
\hline \multirow{2}{*}{$N=100000$} & 3.1e-07 & $2.002 \mathrm{e}-06$ & $8.24 \mathrm{e}-07$ & $2.446 \mathrm{e}-06$ \\
\hline & 4.3e-08 & $4.553 \mathrm{e}-06$ & $4.490 \mathrm{e}-06$ & $5.261 \mathrm{e}-06$ \\
\hline \multirow{2}{*}{$N=1000000$} & $2.627 e-8$ & $2.241 \mathrm{e}-7$ & $6.521 \mathrm{e}-8$ & $4.726 \mathrm{e}-02$ \\
\hline & $3.127 e-9$ & 6.363e-7 & 3.933e-7 & $1.222 \mathrm{e}-01$ \\
\hline
\end{tabular}

\section{Conclusions}

On basis of tent map entropy properties, this work especially compared the entropy properties of several classical maps, and by experiments here got the conclusions as follow:

(1) The entropy values of several classical maps have no relationship to the system initial value, have some relation to the system parameters. This means the system entropies are non-sensitive to initial values, and entropy value of Chaos system with certain parameter is basically confirmed.

(2) The experiment results proved that in several classical Chaos system maps, tent map will convergent to the entropy limit faster,which means when the sequence length is certain, the ergodic of tent map sequence is more uniformed, with small fluctuation. So that when applying it in other areas, the choice of tent map is theorical.

\section{Acknowledgments}

This work was financially supported by National Natural Science Foundation of China (11401284), Doctoral Research Foundation of the Ministry of Education (20132121110009); Liaoning Provincial Department of Education Foundation (L2015108)

\section{References}

[1] Tsuda,I.Information processing in chaotic dynamical system[J].Jour.Fuzzy Soc.Jap, 1993,4(2):220-228.

[2] Choi C,Lee J.Chaotic local search algorithm[J].Artificial Life \& Robotics,2003,2(1):41-47

[3] Wang Zicai,Zhang Tong et al.Simulated annealing optimization method based on chaotic variables [J].control and decision making,1999,40 (13):381-384.

[4] Liu Aijun, Yang Yu et al. Study and application of chaotic simulated annealing particle swarm optimization [J]. Journal of Zhejiang University,2013,47 (10):1722-1730.

[5] Zhang Jinliang,Tan Zhongfu.Hybrid forecasting method of chaotic time series [J].systems engineering theory and practice,2013,33(3):763-769.

[6] Yang Shaoqing et al. A robust algorithm for the estimation of the maximum exponent [J].Acta phys Sinica,2000,49(04):0636-05. 
[7] Yao Minghai, Qi Donglian et al. Study on the control of discrete chaotic system based on the exponent[J].control and decision making,2002,02,0171-04.

[8] Zhang Chuntao, Ma Trinidad. Information entropy optimization of phase space reconstruction of chaotic time series prediction based on [J].Journal of physics,2010,59(11): 7623-07.

[9] Sun Kehui,he Shaobo et al.Analysis of spectral entropy complexity of chaotic pseudorandom sequences[J].Journal of physics,2013,62(01): 010501-1-8

[10] He Tao,Wang y ou.Prediction of chaotic time series based on Fractal self affine [J].Acta phys Sinica,2007,56 (02):0693-08.

[11] Pan Xinyu, study on entropy properties of.Logistic chaotic system [J].Acta phys Sinica,2012,61 (20):20504-1-5. 